\title{
A Computational Model for Deducting of Prickle Noise From Satellite Galaxy Images
}

\author{
W.Jai Singh, M.Duraisamy
}

\begin{abstract}
Images are intended to exhibit vital information and play an important role in technology and innovation. During their discovery, the main drawback of digital image is the level of noise and depletion. This article introduces a predictive model for satellite galaxy images to infer dull throb noise.The existing filtering techniques for image noise are presented and perhaps an approach is adopted by ascribing variability of pixel values to stuck up cells to discern dull throb noise. Approaches to image reconstruction can preserve image details while suppressing noise from the prickle.This technique's working standard is implemented and reviewed using MATLAB with visualization effects. Outcomes of experiments are compared with indicators of image quality.
\end{abstract}

Keywords: - Prickle Noise, Deduction of image noise, Parameters of image quality

\section{INTRODUCTION}

The focus of Digital Image Processing is to upgrade pictographic information for human imagination and processing of visual data for storage, interaction and representation. Renovation of images from corrupted images is a standard issue in the image processing domain.

Noise may arise in three specific ways in an image, (i) If the picture is scanned from a photograph, the dark matter of the film is a cause of noise. Noise can also be the result of the pictures being destroyed or the scanner itself can create it.(ii) If the picture is obtained specifically in a digital format, noise may be introduced by the data capture mechanism (such as a CCD sensor).(iii) Noise can also be generated by computerized propagation of image data, light intensity and sensor temperature.

The deconvolution picture arena is preoccupied with rebuilding or evaluating the uninhabited picture from an unclear and noisy image [1].

Noise decovery and deduction play a vital role in image reconstruction.Assessing the noise level from a unique image is an immense task, so we need to classify whether regional image variations are due to colour, texture lighting variability from the image itself, or noise variations.Image renovation by eliminating noisy pixels increases the image's value. A corrupted image can be refurbished by procedure, which acknowledges noisy pixels throughout the image [2].

This paper is planned as follows: Section 2 is a brief analysis of the model of depletion and compulsion noise Section 3 describes the Mean Filtrate Method (MFM) and Adaptive Median Filtrate Method (AMFM) and details the

Revised Manuscript Received on August 14, 2019.

W.Jai Singh, Assistant Professor (SRG), Dept. of Computer Applications, Kumaraguru College of Technology, Coimbatore, Tamil Nadu, India. (E- Mail: jaisinghw@gmail.com)

M.Duraisamy, Assistant Professor, Department of Computer Science, Thiruvalluvar University College of Arts and Science, Tirupathur - 635 901, Tamil Nadu, India. (E- Mail: duraimca78@gmail.com) proposed Pixel Phenotypic differences Sharing Algorithm (PDSA) method in section 4.Experimental evaluation is conducted in section 5 and concluded in section 6 .

\section{DIGITAL IMAGE HUMILIATION}

Digital Image Embarrassment is a method that acts on a $\mathrm{p}(\mathrm{x}, \mathrm{y})$ interface image via $\mathrm{H}$ humiliation and $\mathrm{n}(\mathrm{x}, \mathrm{y})$ additive noise. It results in tainted $\mathrm{h}(\mathrm{x}, \mathrm{y})$ picture as follows:

$$
\mathrm{h}(\mathrm{a}, \mathrm{b})=\mathrm{s}(\mathrm{a}, \mathrm{b}) \times \mathrm{q}(\mathrm{a}, \mathrm{b})+\mathrm{m}(\mathrm{a}, \mathrm{b})
$$

Where $s(a, b)$ represents the humiliation function spatially and the character ' $\mathrm{x}$ ' indicates intricacy.

\section{STUDY OF DENOISING STRATEGIES}

Linear noise filters tend to skew sharp edges, demolish lines, and other appropriate image specifics. The method of non-linear noise filtering has double phase filtering. The pixels are identified as unnatural or natural pixels in the first phase, and the unnatural pixel is rinsed in the second phase, retaining the natural pixel value.

The Mean Filtrate Method is applied monotonically to the image's whole pixels without characterizing the image as unnatural or natural pixels. Since all the frames in the image are used with these methods, Natural pixels are filtered, which means that these techniques do not work to remove thoughtless noise.
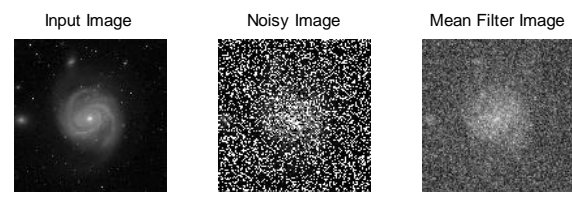

Fig. 1 Mean filtrate rehabilitation method

The Standard Median Filtrate uses its median value to replace the plundered pixel and is capable of eradicating thoughtless noise while preserving the edges. It is also called a filter for order statistics. This method's main notion is to record the frequency pixel by pixel and swap each entry with the neighboring pixel median.
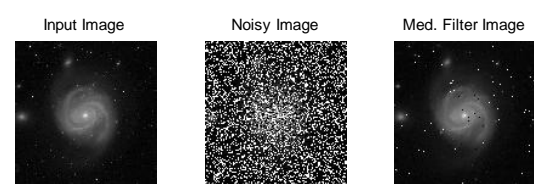

Fig. 2 Standard median filtrate rehabilitation

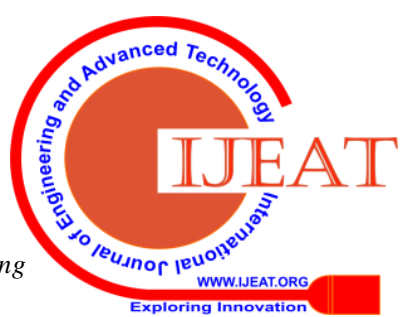




\section{A COMPUTATIONAL MODEL FOR DEDUCTING OF PRICKLE NOISE FROM SATELLITE GALAXY IMAGES}

A major advantage of the standard median filtrate technique is that with increasingly massive amounts it can remove the side effect of input noise values. A four-sided window of scale $2 n+1$, where $n$ goes from 1 to $M$, is applied in the standard median filtrate technique to filter the middle point pixel. First, the pixels in the window are structured and the middle point pixel is altered to the organized sequence's median value.

In order to eliminate thoughtless noise, the adaptive median filtrate technique also applies noise validation and solvent extraction procedures. The window scale used to screen image pixels is highly adaptable in nature, i.e. if the specified constraint is not met, the window size will be reinforced. If the constraint is met, the pixel will be screened using the window median.

Adaptive median filtrate techniques are typically used in the removing impurities of images that have been de-noised by more than 20 percent with noise depth.
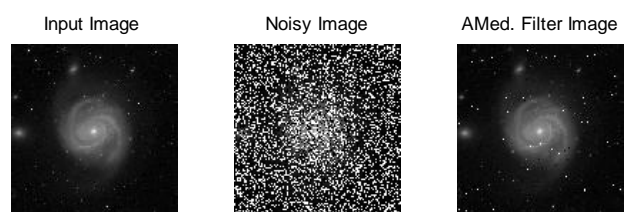

Fig. 3 Adaptive median filtrate restoration

\section{PIXEL DISSIMILARITY SHARING} ALGORITHM - PDSA

Image reconstruction techniques are usually mathematical modelling of the reverse process of embarrassment and slandering to restore the old image. Pixel Dissimilarity Sharing algorithm (PDSA) is performed in the proposed method to restore the image. There are two stages in the procedure.

Stage-I: By beholding at the pixel value, it routes the first column of corrupted image pixels to choose whether or not the pixel is infected. If the pixel value is between the lower limit (0) and the maximum value (255), the pixel is preserved; otherwise the adjacent uncorrupted pixel value is exchanged.

Stage-II: In this stage, Pixel Dissimilarity sharing algorithm (PDSA) is actually employed. Here the procedure is executed by row wise. It begins with pixel location $\left(\mathrm{I}_{\mathrm{i}, \mathrm{j}+1}\right)$ and set the counter to 0 . If the pixel is normal, it will travel to the next pixel location $\left(\mathrm{I}_{\mathrm{i}, \mathrm{j}+2}\right)$, otherwise it will increase the counter and jump to the next move. Until the unspoiled pixel is identified, the above step is reiterated.

Now counter has between two ordinary pixels the number of tainted pixels. These two frames are referred to as pixels of source and destination. The source and destination pixel variance is acquired and the counter value divides this variance. This result is the right pixel deviation value that tends between the pixels of source and destination.

The next step leaps from the source pixel's next location and finishes with the destination pixel's previous location. If the value of the origin pixel is below the value of the destination pixel, the variance is added with the value of the source pixel else the value is subtracted from the value of the destination pixel and the value of the counter is lowered by one at the original location of the repetition. The cycle goes on until the counter value is zero and all the tainted image pixels are tested.
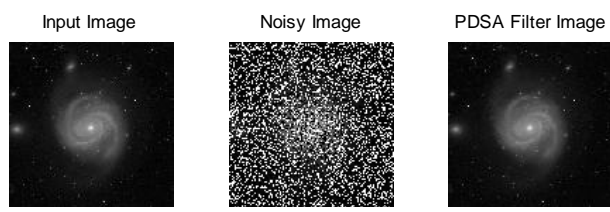

Fig. 4 Pixel-dissimilarity sharing (PDSA) filtration refurbishment

\section{PERFORMANCE EVALUATION RESULTS}

Based on image quality metrics such as Mean Square Error Value (MSEV), Peak Signal to Noise Ratio Value (PSNRV) and Structural Similarity Index Metric Value (SSIMV), the above methods of restoration are calculated.

The above procedures are all performed in a 512 x 512pixel galaxy image. Noises of desire are attached to the normal image and their quality is predicted.
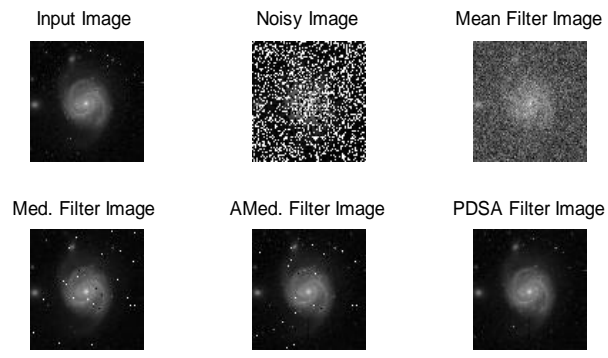

Fig. 5 Imitation meanings for a $50 \%$ noise thickness galaxy image

We estimate and compare the values of MSEV, PSNRV and SSIMV with PDSA values. These results are displayed in graphical notes.

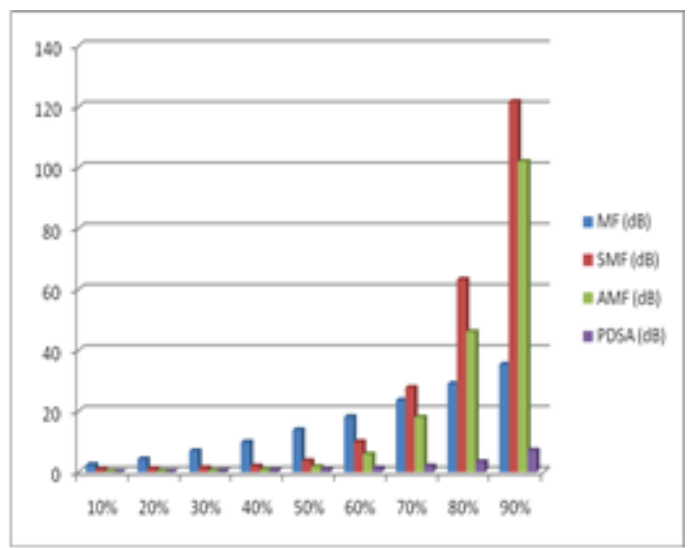

Fig. 6 Mean Square Error Values 


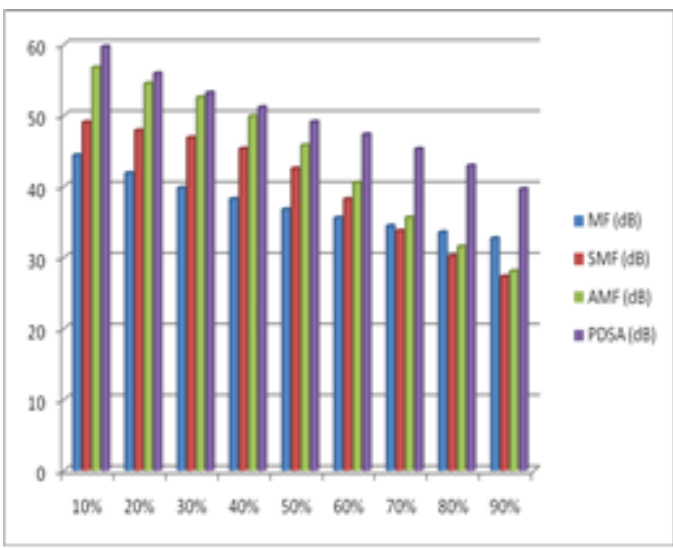

Fig. 7 PSNR Metric Values

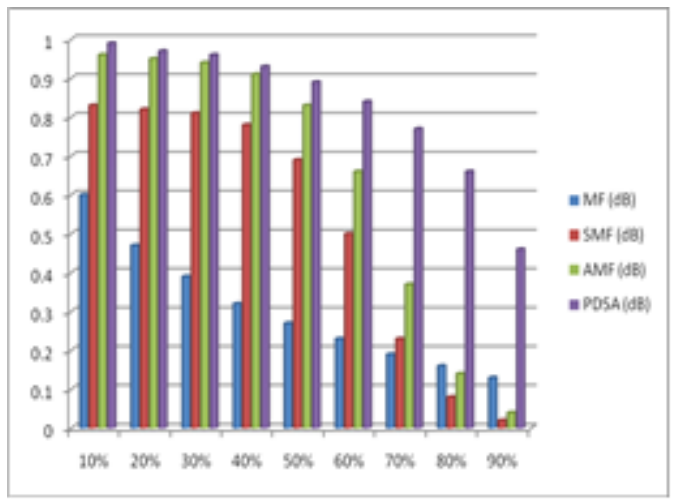

Fig. 8 SSIM Metric Values

It is recommended that the values obtained from the above diagrams perform better than other techniques by Pixel Dissimilarity Sharing Algorithm (PDSA).

\section{CONCLUSION}

Discussed the different image filtering schemes (MFM, SMFM, AMFM) and suggested a new strategy in the paper. The proposed PDSA method yields better results. Based on image quality variables such as MSEV, PSNRV and SSIMV, the reconstructed images were compared.

The values based on the technique of adaptive median filtrate (AMFM) yielded better results than two other techniques. The Pixel Dissimilarity Sharing Algorithm (PDSA) gives decent visual transparency and better results than the AMFM method.

\section{REFERENCES}

1. Anji Reddy M., Hari Shankar Y., Digital Image Processing, BS Publications, 2009.

2. Ben Hamza, P. Luque, J. Martinez, and R. Roman, "Removing noise and preserving details with relaxed median filters," Journal of Mathematical Image Vision, Vol. 11, No. 2, pp. 161-177, October 1999.

3. Chin-Chen Chang, Taichung, and Chih-Ping Hsieh, "An Adaptive Median Filter for Image Denoising", Intelligent Information Technology Application, IEEE, pp. 346-350, ISBN 978-0-7695-3497-8, Shanghai, 2008.

4. Duraisamy. M., Logeswari T., A Template Design Mechanism for CNN Segmentation to Identify Cancer Region on Medical Images, International Journal of Applied Environmental Sciences, Vol.10, No.5 (2015), pp. 1615-1632.

5. Hani M. Ibrahem, An Efficient and simple switching filter for removal of high density salt and pepper noise, International
Journal of Image Graphics and signal processing, Vol. 12 (2013), pp.1-8.

6. E.Jebamalar Leavline and D.Asir Antony Gnana Singh, Salt and Pepper Noise Detection and Removal in Gray Scale Images: An Experimental Analysis, International Journal of Signal Processing, Image Processing and Pattern Recognition, Vol.6, No.5 (2013), pp.343-352.

7. K. S. Srinivasan and D. Ebenezer, "A new fast and efficient decision based algorithm for removal of high density impulse noise," IEEE signal process, Lett. Vol. 14, No. 3, pp. 189-192, March 2007.

8. Poorna Banerjee Dasgupta, Analytical Comparison of Noise Reduction Filters for Image Restoration using SNR Estimation, International Journal of Computer Trends and Technology, Vol.17, No.3 (2014), pp. 121-124.

9. Trapti Soni and Narendra Rathor, Removal of High Density Impulse Noise using Efficient Median filter for Digital Image, International Journal of Computer Applications, Vol.115, No.5 (2015), pp. 25-31.

10. Xudong Jiang, Iterative Truncated Arithmetic Mean Filter and Its Properties, IEEE Transactions on Image Processing, Vol.21, No.4 (2012), pp. 1537-1547. 